\title{
Oscillation constants for half-linear difference equations with coefficients having mean values
}

Petr Hasil and Michal Veselý

\section{"Correspondence:}

michal.vesely@mail.muni.cz Department of Mathematics and Statistics, Masaryk University, Kotlářská 2, Brno, 611 37, Czech Republic

\begin{abstract}
We investigate second order half-linear Euler type difference equations whose coefficients have mean values. We show that these equations are conditionally oscillatory and we explicitly identify the corresponding oscillation constants given by the coefficients. Our results generalize the known ones concerning equations with positive constant, periodic, or (asymptotically) almost periodic coefficients. We also demonstrate the obtained results on examples and we give corollaries. In particular, we get new results even for linear difference equations.
\end{abstract}

MSC: 39A10; 39A21

Keywords: half-linear difference equation; oscillation theory; conditional oscillation; oscillation constant; Euler equation; Riccati technique

\section{Introduction}

This paper is devoted to the oscillatory properties of the half-linear difference equations

$$
\Delta\left[r_{k} \Phi\left(\Delta x_{k}\right)\right]+c_{k} \Phi\left(x_{k+1}\right)=0, \quad \Phi(x)=|x|^{p-1} \operatorname{sgn} x, \quad p>1
$$

where $r_{k}$ is positive for all considered $k$. Throughout the whole paper, we consider integers $k \geq a$ for a sufficiently large number $a \in \mathbb{N}$. For the reader's convenience, we use the notation $\mathbb{N}_{a}:=\{n \in \mathbb{N}: n \geq a\}$ for $a \in \mathbb{N}$. Our aim is to find explicit oscillation constants for all equations (1.1) from a large class of equations.

To recall the notion of the (critical) oscillation constant, we consider (1.1) with

$$
\left\{c_{k}\right\}_{k \in \mathbb{N}_{a}} \equiv\left\{\frac{\gamma s_{k}}{(k+1)^{(p)}}\right\}_{k \in \mathbb{N}_{a}},
$$

i.e., in the form

$$
\Delta\left[r_{k} \Phi\left(\Delta x_{k}\right)\right]+\frac{\gamma s_{k}}{(k+1)^{(p)}} \Phi\left(x_{k+1}\right)=0
$$

(0) 2015 Hasil and Vesely. This article is distributed under the terms of the Creative Commons Attribution 4.0 International License (http://creativecommons.org/licenses/by/4.0/), which permits unrestricted use, distribution, and reproduction in any medium, provided you give appropriate credit to the original author(s) and the source, provide a link to the Creative Commons license, and indicate if changes were made. 
where $\gamma \in \mathbb{R}$ and $k^{(p)}$ stands for the generalized power function (also called the falling factorial power) given by

$$
k^{(p)}=\frac{\Gamma(k+1)}{\Gamma(k+1-p)}, \quad \Gamma(x)=\int_{0}^{\infty} \mathrm{e}^{-s} s^{x-1} \mathrm{~d} s, \quad x>0 .
$$

For details about $k^{(p)}$, see, e.g., Chapter 2 in [1]. Equation (1.2) is said to be conditionally oscillatory if there exists a constant $\Lambda>0$ such that (1.2) is oscillatory for $\gamma>\Lambda$ and nonoscillatory for $\gamma<\Lambda$. The constant $\Lambda$ (which is dependent on coefficients) is called the (critical) oscillation constant of (1.2).

Concerning the conditional oscillation of the studied difference equations, the first result comes from [2], where the equation

$$
\Delta^{2} x_{k}+\frac{\gamma}{(k+1) k} x_{k+1}=0
$$

was proved to be conditionally oscillatory with the oscillation constant $\Lambda=1 / 4$. Equations with non-constant coefficients were analyzed in [3]. In [4], the conditional oscillation of the linear equation

$$
\Delta\left(r_{k} \Delta x_{k}\right)+\frac{\gamma s_{k}}{(k+1) k} x_{k+1}=0
$$

with almost periodic coefficients was obtained. In [5], this result was generalized for halflinear equations of the form (1.2) with positive asymptotically almost periodic sequences $\left\{r_{k}\right\},\left\{s_{k}\right\}$.

Since the main result of [5] is one of the basic motivations for the research presented here, we reformulate it as follows. We remark that the symbol $M(\cdot)$ stands for the mean values of the indicated sequences clarified in Definition 2 below and that the definition of asymptotic almost periodicity is mentioned in Definition 4 below.

Theorem 1 Let $\gamma \in \mathbb{R}$ be given and let $\left\{r_{k}\right\}_{k \in \mathbb{N}_{a}}$ and $\left\{s_{k}\right\}_{k \in \mathbb{N}_{a}}$ be arbitrary positive asymptotically almost periodic sequences such that

$$
\inf \left\{r_{k} ; k \in \mathbb{N}_{a}\right\}>0, \quad \limsup _{k \rightarrow \infty} s_{k}>0 .
$$

Let

$$
\Lambda:=\left(\frac{p-1}{p}\right)^{p}\left[M\left(\left\{r_{k}^{\frac{1}{1-p}}\right\}\right)\right]^{1-p}\left[M\left(\left\{s_{k}\right\}\right)\right]^{-1}
$$

If $\gamma>\Lambda$, then (1.2) is oscillatory. If $\gamma<\Lambda$, then (1.2) is non-oscillatory.

Our second basic motivation comes from the continuous case. We begin with a short historical summary of the studied problem. The first attempt to treat the conditional oscillation can be found in [6], where the oscillation constant $\Lambda=1 / 4$ was identified for the equation

$$
x^{\prime \prime}+\frac{\gamma}{t^{2}} x=0
$$


In $[7,8]$, this result was extended to linear differential equations

$$
\left(r(t) x^{\prime}\right)^{\prime}+\frac{\gamma s(t)}{t^{2}} x=0
$$

with positive $\alpha$-periodic coefficients $r, s$ and with the oscillation constant

$$
\Lambda=\frac{\alpha^{2}}{4}\left(\int_{0}^{\alpha} \frac{\mathrm{d} \tau}{r(\tau)}\right)^{-1}\left(\int_{0}^{\alpha} s(\tau) \mathrm{d} \tau\right)^{-1} .
$$

For more general results, we refer, e.g., to [9-12].

The concept of the conditional oscillation was transferred to half-linear differential equations in [13] (see also [14]), where the oscillation constant

$$
\Lambda=\left(\frac{p-1}{p}\right)^{p}
$$

was obtained for the equation

$$
\left[\Phi\left(x^{\prime}\right)\right]^{\prime}+\frac{\gamma}{t^{p}} \Phi(x)=0
$$

During the last years, the conditional oscillation was resolved for half-linear differential equations with positive periodic coefficients (see [15]) and half-linear equations with more general coefficients (see, e.g., [16-19]). Along this line, the most relevant result for our research comes from [20]. Since it is our second main motivation, we mention the main result of [20] in full. We remark that $\mathbb{R}_{a}:=[a, \infty)$ and $M(\cdot)$ also stands for the mean values of considered functions (for details, see [20]).

Theorem 2 Let $r: \mathbb{R}_{a} \rightarrow \mathbb{R}$ be a continuous function, for which mean value $M\left(r^{\frac{1}{1-p}}\right)$ exists and for which we have

$$
0<\inf _{t \in \mathbb{R}_{a}} r(t) \leq \sup _{t \in \mathbb{R}_{a}} r(t)<\infty
$$

and let $s: \mathbb{R}_{a} \rightarrow \mathbb{R}$ be a continuous function having mean value $M(s)$. Let

$$
\Gamma:=\left(\frac{p-1}{p}\right)^{p}\left[M\left(r^{\frac{1}{1-p}}\right)\right]^{1-p}=\left(\frac{p-1}{p}\right)^{p}\left[\lim _{t \rightarrow \infty} \frac{1}{t} \int_{a}^{a+t} r^{\frac{1}{1-p}}(\tau) \mathrm{d} \tau\right]^{1-p} .
$$

Consider the equation

$$
\left[r(t) \Phi\left(x^{\prime}\right)\right]^{\prime}+\frac{s(t)}{t^{p}} \Phi(x)=0
$$

Equation (1.3) is oscillatory if $M(s)>\Gamma$, and non-oscillatory if $M(s)<\Gamma$.

In this paper, we intend to generalize Theorem 1 to the case that the coefficients have mean values and the second coefficient can change sign. It means that our aim is to prove the discrete counterpart of Theorem 2. For this purpose, we improve the method from [5]. Since we study equations with coefficients from more general classes, we have to prove 
some new auxiliary results and inequalities (especially, we need Lemmas 1 and 2 below). Note that we partially apply the processes used in [5] (see the proof of Theorem 5 below, where it is explicitly mentioned).

The paper is organized as follows. In the next section, we state the necessary background and we recall the so-called Riccati technique, which is essential for our investigation. In Section 3, the reader can find preparatory lemmas, results, and corollaries. In Section 4, we collect illustrative examples.

Throughout the paper, we will consider an arbitrarily given number $p>1$ from the shape of $\Phi(x)=|x|^{p-1} \operatorname{sgn} x$. We will use the standard notation of conjugated numbers $p, q$, which means that $q>1$ will denote the real number satisfying

$$
\frac{1}{p}+\frac{1}{q}=1, \quad \text { i.e. }, p+q=p q .
$$

\section{Preliminaries}

In this section, we mention the needed background concerning the oscillation theory of half-linear difference equations. For more details, we refer to Chapter 3 in [21] and Chapter 8 in [22] with references cited therein. In addition, we recall the concept of mean values which is necessary to find general oscillation constants. We also state the concept of the (adapted) half-linear Riccati equation which is the main tool in our investigation.

At first, we recall the basic notions from the oscillation theory of the half-linear equation

$$
\Delta\left[r_{k} \Phi\left(\Delta x_{k}\right)\right]+c_{k} \Phi\left(x_{k+1}\right)=0,
$$

where $r_{k}>0$ for all considered $k \in \mathbb{N}_{a}$. An interval $(l, l+1], l \in \mathbb{N}_{a}$, contains the generalized zero of a solution $\left\{x_{k}\right\}$ of (2.1) if $x_{l} \neq 0$ and $x_{l} x_{l+1} \leq 0$. We say that (2.1) is disconjugate on a set $\{l, l+1, \ldots, l+n\}$ if any solution of $(2.1)$ has at most one generalized zero on $(l, l+n+1]$ and a solution $\left\{\tilde{x}_{k}\right\}$ given by the initial value $\tilde{x}_{l}=0$ has no generalized zero on $(l, l+n+1]$. Otherwise, (2.1) is called conjugate on $\{l, l+1, \ldots, l+n\}$. Now we can formulate the following definition.

Definition 1 Equation (2.1) is called non-oscillatory if there exists $l \in \mathbb{N}$ with the property that (2.1) is disconjugate on $\{l, l+1, \ldots, l+n\}$ for all $n \in \mathbb{N}$. In the opposite case, (2.1) is called oscillatory.

The Sturm type separation theorem (see, e.g., Theorem 3.3.6 in [21]) enables us to give Definition 1, because the oscillation of an arbitrary non-zero solution of (2.1) implies the oscillation of all solutions of (2.1). We will also use a consequence of the Sturm type comparison theorem. We mention only the form that is suitable for our purpose.

Theorem 3 Let $\left\{y_{k}\right\}_{k \in \mathbb{N}_{a}},\left\{Y_{k}\right\}_{k \in \mathbb{N}_{a}},\left\{z_{k}\right\}_{k \in \mathbb{N}_{a}},\left\{Z_{k}\right\}_{k \in \mathbb{N}_{a}}$ be sequences satisfying the inequalities $y_{k} \geq Y_{k}>0, Z_{k} \geq z_{k}$ for all sufficiently large $k$. Let us consider the equations

$$
\begin{aligned}
& \Delta\left[y_{k} \Phi\left(\Delta x_{k}\right)\right]+z_{k} \Phi\left(x_{k+1}\right)=0, \\
& \Delta\left[Y_{k} \Phi\left(\Delta x_{k}\right)\right]+Z_{k} \Phi\left(x_{k+1}\right)=0 .
\end{aligned}
$$

If (2.3) is non-oscillatory, then (2.2) is non-oscillatory as well. 
Proof The theorem follows, e.g., from Theorem 3.3.5 in [21].

To obtain explicit oscillation constants, we need the definition of the mean value of a sequence.

Definition 2 Let a sequence $\left\{f_{k}\right\}_{k \in \mathbb{N}_{a}} \subset \mathbb{R}$ be given and let the limit

$$
M\left(\left\{f_{k}\right\}\right):=\lim _{n \rightarrow \infty} \frac{1}{n} \sum_{k=i}^{n+i-1} f_{k}
$$

be finite and exist uniformly with respect to $i \in \mathbb{N}_{a}$. The number $M\left(\left\{f_{k}\right\}\right)$ introduced in (2.4) is called the mean value of $\left\{f_{k}\right\}$.

An important class of sequences having mean values is formed by asymptotically almost periodic sequences (see also [5]). Hence, we formulate the next definitions.

Definition 3 A sequence $\left\{f_{k}\right\}_{k \in \mathbb{Z}} \subset \mathbb{R}$ is called almost periodic if, for any $\varepsilon>0$, there exists $P(\varepsilon) \in \mathbb{N}$ such that any set of the form $\{i, i+1, \ldots, i+P(\varepsilon)-1\} \subset \mathbb{Z}$ contains an integer $l$ for which $\left|f_{k}-f_{k+l}\right|<\varepsilon, k \in \mathbb{Z}$.

Definition 4 We say that a sequence $\left\{f_{k}\right\}_{k \in \mathbb{N}_{a}} \subset \mathbb{R}$ is asymptotically almost periodic if there exists a pair of sequences $\left\{f_{k}^{1}\right\}_{k \in \mathbb{Z}},\left\{f_{k}^{2}\right\}_{k \in \mathbb{N}_{a}} \subset \mathbb{R}$ such that $\left\{f_{k}^{1}\right\}$ is almost periodic, $\left\{f_{k}^{2}\right\}$ satisfies $\lim _{k \rightarrow \infty} f_{k}^{2}=0$, and $\left\{f_{k}\right\}_{k \in \mathbb{N}_{a}} \equiv\left\{f_{k}^{1}+f_{k}^{2}\right\}_{k \in \mathbb{N}_{a}}$.

Finally, we describe the half-linear Riccati equation and its adapted version. Using the so-called Riccati substitution

$$
w_{k}=r_{k} \Phi\left(\frac{\Delta x_{k}}{x_{k}}\right)
$$

to (2.1), we obtain the associated Riccati equation

$$
\Delta w_{k}+c_{k}+w_{k}\left(1-\frac{r_{k}}{\Phi\left[\Phi^{-1}\left(r_{k}\right)+\Phi^{-1}\left(w_{k}\right)\right]}\right)=0
$$

where $\Phi^{-1}$ denotes the inverse function of $\Phi$, i.e., $\Phi^{-1}(x)=|x|^{q-1} \operatorname{sgn} x$. Under the condition $w_{k}+r_{k}>0$, we can express (see Lemma 3.2.6, $\left(\mathrm{I}_{8}\right)$ in [21])

$$
w_{k}\left(1-\frac{r_{k}}{\Phi\left[\Phi^{-1}\left(r_{k}\right)+\Phi^{-1}\left(w_{k}\right)\right]}\right)=\frac{(p-1)\left|w_{k}\right|^{q}\left|\beta_{k}\right|^{p-2}}{\Phi\left[\Phi^{-1}\left(r_{k}\right)+\Phi^{-1}\left(w_{k}\right)\right]},
$$

where $\beta_{k}$ is between $\Phi^{-1}\left(r_{k}\right)$ and $\Phi^{-1}\left(r_{k}\right)+\Phi^{-1}\left(w_{k}\right)$; i.e., for $w_{k}+r_{k}>0$, we have the Riccati equation (2.5) associated to (2.1) in the form

$$
\Delta w_{k}+c_{k}+\frac{(p-1)\left|w_{k}\right|^{q}\left|\beta_{k}\right|^{p-2}}{\Phi\left[\Phi^{-1}\left(r_{k}\right)+\Phi^{-1}\left(w_{k}\right)\right]}=0
$$

The following theorem is typically known as the Riccati method. It shows the way in which the non-oscillation of (2.1) is connected to the solvability of (2.6). 
Theorem 4 Equation (2.1) is non-oscillatory if and only if there exist an integer $b$ and $a$ sequence of $w_{k}$ which solves (2.6) and satisfies $w_{k}+r_{k}>0$ for $k \in \mathbb{N}_{b}$.

Proof The theorem is a consequence of the well-known roundabout theorem (see, e.g., Theorem 3.3.4 in [21] or directly Theorem 8.2.5 in [22]).

Taking into account the second substitution

$$
\zeta_{k}=-k^{(p-1)} w_{k}
$$

together with the Riccati equation (2.6), we obtain the adapted Riccati equation associated to $(2.1)$ as

$$
\Delta \zeta_{k}=\frac{1}{k-p+2}\left[(p-1) \zeta_{k}+(k+1)^{(p)} c_{k}+\frac{(k+1)(p-1)\left|\beta_{k}\right|^{p-2}\left|\zeta_{k}\right|^{q}}{\left[k^{(p-1)}\right]^{q-1} \Phi\left[\Phi^{-1}\left(r_{k}\right)+\Phi^{-1}\left(-\frac{\zeta_{k}}{k^{(p-1)}}\right)\right]}\right],
$$

where $\beta_{k}$ is between $\Phi^{-1}\left(r_{k}\right)$ and $\Phi^{-1}\left(r_{k}\right)+\Phi^{-1}\left(-\frac{\zeta k}{k^{(p-1)}}\right)$.

In fact, we will consider (2.1) in the form

$$
\Delta\left[r_{k} \Phi\left(\Delta x_{k}\right)\right]+\frac{s_{k}}{(k+1)^{(p)}} \Phi\left(x_{k+1}\right)=0,
$$

where sequence $\left\{r_{k}\right\}_{k \in \mathbb{N}_{a}}$ has mean value $M\left(\left\{r_{k}^{1-q}\right\}\right)=1$ and

$$
0<r^{-}:=\inf _{k \in \mathbb{N}_{a}} r_{k} \leq \sup _{k \in \mathbb{N}_{a}} r_{k}=: r^{+}<\infty
$$

and where sequence $\left\{s_{k}\right\}_{k \in \mathbb{N}_{a}}$ has a positive mean value, i.e., $M\left(\left\{s_{k}\right\}\right)>0$. Therefore, we will deal with the Riccati equation associated to (2.9) in the form (see (2.6))

$$
\Delta w_{k}+\frac{s_{k}}{(k+1)^{(p)}}+\frac{(p-1)\left|w_{k}\right|^{q}\left|\beta_{k}\right|^{p-2}}{\Phi\left[\Phi^{-1}\left(r_{k}\right)+\Phi^{-1}\left(w_{k}\right)\right]}=0
$$

and with the adapted Riccati equation (see (2.8))

$$
\Delta \zeta_{k}=\frac{1}{k-p+2}\left[(p-1) \zeta_{k}+s_{k}+\frac{(k+1)(p-1)\left|\beta_{k}\right|^{p-2}\left|\zeta_{k}\right|^{q}}{\left[k^{(p-1)}\right]^{q-1} \Phi\left[\Phi^{-1}\left(r_{k}\right)+\Phi^{-1}\left(-\frac{\zeta_{k}}{k^{(p-1)}}\right)\right.}\right] .
$$

More precisely, we will study (2.9) using (2.12).

\section{Results}

To prove the main results, we need the following lemmas.

Lemma 1 Let a sequence $\left\{f_{k}\right\}_{k \in \mathbb{N}_{a}} \subset \mathbb{R}$ have mean value $M\left(\left\{f_{k}\right\}\right)$. There exists a number $K\left(\left\{f_{k}\right\}\right)>0$ for which $\left|f_{k}\right|<K\left(\left\{f_{k}\right\}\right), k \in \mathbb{N}_{a}$.

Proof The existence of $M\left(\left\{f_{k}\right\}\right)$ gives $m \in \mathbb{N}$ such that

$$
\left|M\left(\left\{f_{k}\right\}\right)-\frac{1}{m+l} \sum_{k=i}^{i+m+l-1} f_{k}\right|<1, \quad i \in \mathbb{N}_{a}, l \in \mathbb{N} \cup\{0\} .
$$


From (3.1) it follows that

$$
\begin{aligned}
& \left|\frac{1}{m} \sum_{k=i+1}^{i+m} f_{k}-\frac{1}{m} \sum_{k=i}^{i+m-1} f_{k}\right|<2, \quad i \in \mathbb{N}_{a}, \\
& \left|\frac{1}{m+1} \sum_{k=i+1}^{i+m+1} f_{k}-\frac{1}{m+1} \sum_{k=i}^{i+m} f_{k}\right|<2, \quad i \in \mathbb{N}_{a} .
\end{aligned}
$$

Especially,

$$
\begin{aligned}
& \left|f_{i}-f_{m+i}\right|<2 m, \quad i \in \mathbb{N}_{a}, \\
& \left|f_{i}-f_{m+i+1}\right|<2(m+1), \quad i \in \mathbb{N}_{a} .
\end{aligned}
$$

Thus, we have

$$
\left|f_{m+i}-f_{m+i+1}\right|<4 m+2, \quad i \in \mathbb{N}_{a}
$$

Finally, we have

$$
\left|f_{i}-f_{i+1}\right| \leq L\left(\left\{f_{k}\right\}\right):=\max \left\{4 m+2,\left|f_{a}-f_{a+1}\right|, \ldots,\left|f_{m+a-1}-f_{m+a}\right|\right\}, \quad i \in \mathbb{N}_{a}
$$

On the contrary, let us suppose that $\limsup _{k \rightarrow \infty}\left|f_{k}\right|=\infty$. If

$$
f_{i} \geq M\left(\left\{f_{k}\right\}\right)+1+(m-1) L\left(\left\{f_{k}\right\}\right)
$$

for some $i \in \mathbb{N}_{a}$, then

$$
f_{i+j} \geq M\left(\left\{f_{k}\right\}\right)+1, \quad j \in\{0,1, \ldots, m-1\} .
$$

Analogously, if

$$
f_{i} \leq M\left(\left\{f_{k}\right\}\right)-1-(m-1) L\left(\left\{f_{k}\right\}\right)
$$

for some $i \in \mathbb{N}_{a}$, then

$$
f_{i+j} \leq M\left(\left\{f_{k}\right\}\right)-1, \quad j \in\{0,1, \ldots, m-1\} .
$$

Of course, each one of inequalities (3.2), (3.3) gives a contradiction with (3.1) for $l=0$. It means that it suffices to put

$$
K\left(\left\{f_{k}\right\}\right):=\left|M\left(\left\{f_{k}\right\}\right)\right|+1+(m-1) L\left(\left\{f_{k}\right\}\right)
$$

for $m$ from (3.1).

Henceforth, let $m \in \mathbb{N}$ be such that

$$
\frac{3 M\left(\left\{s_{k}\right\}\right)}{2}>\frac{1}{m+l} \sum_{k=i}^{i+m+l-1} s_{k}>\frac{M\left(\left\{s_{k}\right\}\right)}{2}>0, \quad i \in \mathbb{N}_{a}, l \in \mathbb{N} \cup\{0\}
$$


We also put (cf. (2.10))

$$
s^{+}:=\sup _{k \in \mathbb{N}_{a}}\left|s_{k}\right|<\infty
$$

where we use Lemma 1.

Lemma 2 If (2.9) is non-oscillatory, then there exist $L \in \mathbb{N}$ and a negative solution $\left\{\zeta_{k}\right\}_{k \in \mathbb{N}_{L}}$ of (2.12) such that

$$
\lim _{k \rightarrow \infty} \frac{\zeta_{k}}{k^{(p-1)}}=0
$$

Proof Considering Theorem 4, the non-oscillation of (2.9) implies that there exist $L \in \mathbb{N}$ and a solution $\left\{w_{k}\right\}_{k \in \mathbb{N}_{L}}$ of (2.11) such that $w_{k}+r_{k}>0$ for $k \geq L$. Considering (2.7), it gives the solution $\left\{\zeta_{k}\right\}_{k \in \mathbb{N}_{L}} \equiv\left\{-w_{k} k^{(p-1)}\right\}_{k \in \mathbb{N}_{L}}$ of (2.12). We show that this solution $\left\{\zeta_{k}\right\}$ is negative and satisfies (3.6).

In fact, we show that the sequences $\left\{w_{m k}\right\},\left\{w_{m k+1}\right\}, \ldots,\left\{w_{m k+m-1}\right\}$ are decreasing for sufficiently large $k$ and tend to zero. Let $j \in\{0,1, \ldots, m-1\}$ be arbitrarily given. From (2.11), we have

$$
w_{(k+1) m+j}-w_{m k+j}=-\sum_{i=m k+j}^{(k+1) m+j-1} \frac{s_{i}}{(i+1)^{(p)}}-\sum_{i=m k+j}^{(k+1) m+j-1} \frac{(p-1)\left|w_{i}\right|^{q}\left|\beta_{i}\right|^{p-2}}{\Phi\left[\Phi^{-1}\left(r_{i}\right)+\Phi^{-1}\left(w_{i}\right)\right]}
$$

for all considered $k$. Since $\left\{s_{k}\right\}$ is bounded (consider Lemma 1) and

$$
\lim _{l \rightarrow \infty} \frac{(l+m)^{(p)}}{(l+1)^{(p)}}=1
$$

using (3.4), we obtain

$$
-\sum_{i=m k+j}^{(k+1) m+j-1} \frac{s_{i}}{(i+1)^{(p)}}<0
$$

for all large $k$. Considering $w_{k}+r_{k}>0$ for $k \geq L$, it is seen that

$$
\Phi\left[\Phi^{-1}\left(r_{k}\right)+\Phi^{-1}\left(w_{k}\right)\right]>0, \quad k \geq L .
$$

From (3.7), (3.8), (3.9), we get $w_{(k+1) m+j}<w_{m k+j}$ for all large $k$. Thus, there exist limits (as real numbers or $-\infty)$

$$
L_{0}:=\lim _{k \rightarrow \infty} w_{m k}, \quad L_{1}:=\lim _{k \rightarrow \infty} w_{m k+1}, \quad \ldots, \quad L_{m-1}:=\lim _{k \rightarrow \infty} w_{m k+m-1} .
$$

Our aim is to prove that $L_{j}=0$ for each $j \in\{0,1, \ldots, m-1\}$. On contrary, let us consider that $L_{j} \neq 0$ for some $j$. Denote $W:=\max _{k \in \mathbb{N}_{L}} w_{k}$.

Let $L_{j}>\varepsilon>0$. We know that

$$
\Phi^{-1}(W)+\Phi^{-1}\left(r^{+}\right) \geq \beta_{k m+j} \geq \Phi^{-1}\left(r_{k m+j}\right) \geq \Phi^{-1}\left(r^{-}\right)>0
$$


and that

$$
\Phi\left[\Phi^{-1}\left(r_{k m+j}\right)+\Phi^{-1}\left(w_{m k+j}\right)\right]<\Phi\left[\Phi^{-1}\left(r^{+}\right)+\Phi^{-1}(W)\right]
$$

for all $k$. Hence (see (3.10)), there exists $B_{j}>0$ with the property that

$$
\left|\beta_{m k+j}\right|^{p-2}>B_{j}, \quad k \geq L
$$

In addition, for large $k$, we have (see (3.7), (3.8), (3.9), (3.11), and (3.12))

$$
\begin{aligned}
w_{(m+1) k+j}-w_{m k+j} & <-\sum_{i=m k+j}^{(k+1) m+j-1} \frac{(p-1)\left|w_{i}\right|^{q}\left|\beta_{i}\right|^{p-2}}{\Phi\left[\Phi^{-1}\left(r_{i}\right)+\Phi^{-1}\left(w_{i}\right)\right]} \\
& <-\frac{(p-1)\left|w_{m k+j}\right|^{q}\left|\beta_{m k+j}\right|^{p-2}}{\Phi\left[\Phi^{-1}\left(r_{m k+j}\right)+\Phi^{-1}\left(w_{m k+j}\right)\right]}<-\frac{(p-1) \varepsilon^{q} B_{j}}{\Phi\left[\Phi^{-1}\left(r^{+}\right)+\Phi^{-1}(W)\right]} .
\end{aligned}
$$

But we obtain the contradiction $L_{j}=-\infty$, because the last term is a negative constant.

Let $L_{j}<-\varepsilon<0$, i.e., $w_{m k+j}<-\varepsilon$ for large $k$. In this case, for large $k$, we have

$$
\begin{aligned}
w_{(m+1) k+j}-w_{m k+j} & <-\sum_{i=m k+j}^{(k+1) m+j-1} \frac{(p-1)\left|w_{i}\right|^{q}\left|\beta_{i}\right|^{p-2}}{\Phi\left[\Phi^{-1}\left(r_{i}\right)+\Phi^{-1}\left(w_{i}\right)\right]} \\
& <-\frac{(p-1)\left|w_{m k+j}\right|^{q}\left|\beta_{m k+j}\right|^{p-2}}{\Phi\left[\Phi^{-1}\left(r_{m k+j}\right)+\Phi^{-1}\left(w_{m k+j}\right)\right]} \\
& <-\frac{(p-1)|\varepsilon|^{q}\left[\Phi^{-1}\left(r_{m k+j}\right)+\Phi^{-1}\left(w_{m k+j}\right)\right]^{p-2}}{\Phi\left[\Phi^{-1}\left(r_{m k+j}\right)+\Phi^{-1}\left(w_{m k+j}\right)\right]} \\
& =-\frac{(p-1)|\varepsilon|^{q}}{\Phi^{-1}\left(r_{m k+j}\right)+\Phi^{-1}\left(w_{m k+j}\right)}<-\frac{(p-1) \varepsilon^{q}}{\Phi^{-1}\left(r^{+}\right)}
\end{aligned}
$$

if $p \geq 2$; and

$$
\begin{aligned}
w_{(m+1) k+j}-w_{m k+j} & <-\frac{(p-1)\left|w_{m k+j}\right|^{q}\left|\beta_{m k+j}\right|^{p-2}}{\Phi\left[\Phi^{-1}\left(r_{m k+j}\right)+\Phi^{-1}\left(w_{m k+j}\right)\right]} \\
& <-\frac{(p-1)|\varepsilon|^{q}\left[\Phi^{-1}\left(r_{m k+j}\right)\right]^{p-2}}{\Phi\left[\Phi^{-1}\left(r_{m k+j}\right)\right]}<-\frac{(p-1) \varepsilon^{q}}{\Phi^{-1}\left(r^{+}\right)}
\end{aligned}
$$

if $p \in(1,2)$. Again, for any $p>1$, we get $L_{j}=-\infty$, which cannot be true, because $w_{k}+r_{k}>0$ for all $k$ and $\left\{r_{k}\right\}$ is bounded.

Altogether, we know that $\left\{w_{k}\right\}$ is positive and

$$
\lim _{k \rightarrow \infty} w_{k}=0
$$

Thus, $\left\{\zeta_{k}\right\}$ is negative and (3.6) follows from (3.13).

We remark that, in the case that the sequence of $s_{k}$ is positive, the statement of Lemma 2 follows from Lemma 1, (v) and Theorem 1 in [23] combined with Lemma 3.5.9 in [21] or with Lemma 8.2.2 in [22]. 
Lemma 3 If there exists a negative solution $\left\{\zeta_{k}\right\}_{k \in \mathbb{N}_{L}}$ of (2.12), then (2.9) is non-oscillatory.

Proof A negative solution $\left\{\zeta_{k}\right\}_{k \in \mathbb{N}_{L}}$ of (2.12) gives $\left\{w_{k}\right\}_{k \in \mathbb{N}_{L}} \equiv\left\{-\zeta_{k} / k^{(p-1)}\right\}_{k \in \mathbb{N}_{L}}$, which is a positive solution of (2.11). Thus, the lemma follows from Theorem 4 .

Applying the above lemmas, we can obtain the announced result. For the reader's convenience, we recall the assumptions on the coefficients.

Theorem 5 Let sequence $\left\{r_{k}\right\}_{k \in \mathbb{N}_{a}}$ have mean value $M\left(\left\{r_{k}^{1-q}\right\}\right)=1$ and satisfy (2.10) and let sequence $\left\{s_{k}\right\}_{k \in \mathbb{N}_{a}}$ have mean value $M\left(\left\{s_{k}\right\}\right)>0$. Then (2.9) is oscillatory for $M\left(\left\{s_{k}\right\}\right)>q^{-p}$ and non-oscillatory for $M\left(\left\{s_{k}\right\}\right)<q^{-p}$.

Proof At first, let us approach the oscillatory part of the theorem. By contradiction, we suppose that $M\left(\left\{s_{k}\right\}\right)>q^{-p}$ and that (2.9) is non-oscillatory. Using Lemma 2, we obtain the existence of a negative solution $\left\{\zeta_{k}\right\}_{k \in \mathbb{N}_{L}}$ of (2.12); i.e., we have

$$
\Delta \zeta_{k}=\frac{1}{k-p+2}\left[(p-1) \zeta_{k}+s_{k}+\frac{(k+1)(p-1) \beta_{k}^{p-2}\left|\zeta_{k}\right|^{q}}{\left[k^{(p-1)}\right]^{q-1} \Phi\left[\Phi^{-1}\left(r_{k}\right)+\Phi^{-1}\left(-\frac{\zeta_{k}}{k^{(p-1)}}\right)\right.}\right], \quad k \geq L,
$$

where

$$
0<\Phi^{-1}\left(r_{k}\right) \leq \beta_{k} \leq \Phi^{-1}\left(r_{k}\right)+\Phi^{-1}\left(-\frac{\zeta_{k}}{k^{(p-1)}}\right), \quad k \geq L .
$$

From Lemma 2 (see (2.10), (3.6), and (3.15)), we also obtain

$$
1 \leq \liminf _{k \rightarrow \infty} \frac{\beta_{k}}{\Phi^{-1}\left(r_{k}\right)} \leq \limsup _{k \rightarrow \infty} \frac{\beta_{k}}{\Phi^{-1}\left(r_{k}\right)} \leq \lim _{k \rightarrow \infty} \frac{\Phi^{-1}\left(r_{k}\right)+\Phi^{-1}\left(-\frac{\zeta_{k}}{k^{(p-1)}}\right)}{\Phi^{-1}\left(r_{k}\right)}=1 .
$$

From (3.16) it follows that

$$
\lim _{k \rightarrow \infty} \frac{\beta_{k}^{p-2} r_{k}^{q-1}}{\Phi\left[\Phi^{-1}\left(r_{k}\right)+\Phi^{-1}\left(-\frac{\zeta k}{k^{(p-1)}}\right)\right]}=\lim _{k \rightarrow \infty} \frac{\left[r_{k}^{q-1}\right]^{p-2} r_{k}^{q-1}}{r_{k}}=\lim _{k \rightarrow \infty} \frac{r_{k}^{2-q} r_{k}^{q-1}}{r_{k}}=1 .
$$

It is well known that

$$
\lim _{k \rightarrow \infty} \frac{k+1}{\left[k^{(p-1)}\right]^{q-1}}=\lim _{k \rightarrow \infty} \frac{k}{\left[k^{(p-1)}\right]^{q-1}}=\lim _{k \rightarrow \infty} \frac{k}{\left[k^{p-1}\right]^{q-1}}=1 .
$$

Thus (see (2.10), (3.17), and (3.18)), we can assume that $L>p-2$ is so large that

$$
\begin{aligned}
\frac{1}{\left(\sqrt{2} r^{+}\right)^{q-1}} & \leq \frac{1}{\left(\sqrt{2} r_{k}\right)^{q-1}} \leq \frac{\beta_{k}^{p-2}}{\Phi\left[\Phi^{-1}\left(r_{k}\right)+\Phi^{-1}\left(-\frac{\zeta_{k}}{k^{(p-1)}}\right)\right]} \\
& \leq\left(\frac{\sqrt{2}}{r_{k}}\right)^{q-1} \leq\left(\frac{\sqrt{2}}{r^{-}}\right)^{q-1}, \quad k \geq L,
\end{aligned}
$$

and that

$$
\left(\frac{1}{\sqrt{2}}\right)^{q-1} \leq \frac{k+1}{\left[k^{(p-1)}\right]^{q-1}} \leq 2^{\frac{q-1}{2}}, \quad k \geq L .
$$


Combining (3.19) and (3.20), we obtain

$$
\frac{(p-1)\left|\zeta_{k}\right|^{q}}{\left[2 r^{+}\right]^{q-1}} \leq \frac{(k+1)(p-1) \beta_{k}^{p-2}\left|\zeta_{k}\right|^{q}}{\left[k^{(p-1)}\right]^{q-1} \Phi\left[\Phi^{-1}\left(r_{k}\right)+\Phi^{-1}\left(-\frac{\zeta_{k}}{k^{(p-1)}}\right)\right]} \leq \frac{2^{q-1}(p-1)\left|\zeta_{k}\right|^{q}}{\left[r^{-}\right]^{q-1}}
$$

for all $k \geq L$.

Considering (3.14) and (3.21), we have (see also (3.5))

$$
\Delta \zeta_{k} \geq \frac{1}{k-p+2}\left[(p-1) \zeta_{k}-s^{+}+\frac{(p-1)\left|\zeta_{k}\right|^{q}}{\left[2 r^{+}\right]^{q-1}}\right], \quad k \geq L
$$

and

$$
\Delta \zeta_{k} \leq \frac{1}{k-p+2}\left[s^{+}+\frac{2^{q-1}(p-1)\left|\zeta_{k}\right|^{q}}{\left[r^{-}\right]^{q-1}}\right], \quad k \geq L .
$$

If

$$
\zeta_{i}<X_{1}:=-2 r^{+}\left[2+\frac{s^{+}}{p-1}\right]^{\frac{1}{q-1}}-1
$$

for some $i \geq L$, then

$$
\zeta_{i}-\frac{s^{+}}{p-1}+\frac{\left|\zeta_{i}\right|^{q}}{\left[2 r^{+}\right]^{q-1}}>\left|\zeta_{i}\right|\left(-1+\left[\frac{\left|\zeta_{i}\right|}{2 r^{+}}\right]^{q-1}-\frac{s^{+}}{p-1}\right)>\left|\zeta_{i}\right|(-1+2)>-X_{1}>0 .
$$

Thus, in this case, we have $\zeta_{i+1}>\zeta_{i}$. Indeed, (3.22) and (3.25) give

$$
\Delta \zeta_{i} \geq \frac{p-1}{i-p+2}\left[\zeta_{i}-\frac{s^{+}}{p-1}+\frac{\left|\zeta_{i}\right|^{q}}{\left[2 r^{+}\right]^{q-1}}\right]>\frac{p-1}{i-p+2}\left|X_{1}\right|>0
$$

if (3.24) is valid. Let us consider the smallest integer $l_{0} \geq L$ such that $\zeta_{0} \geq X_{1}$. Note that such a number $l_{0}$ has to exist because its existence follows from (3.26) and from

$$
\sum_{i=L}^{\infty} \frac{p-1}{i-p+2}\left|X_{1}\right| \geq(p-1) \sum_{j=L-p+2}^{\infty} \frac{1}{j}=\infty
$$

Using (3.22), we have

$$
\zeta_{l_{0}+1} \geq X_{2}:=X_{1}+\frac{1}{l_{0}-p+2}\left[(p-1) X_{1}-s^{+}\right] .
$$

Analogously, one can get that $\zeta_{j+1} \geq X_{2}$ if $\zeta_{j} \geq X_{1}$ for some $j \geq l_{0}$. Hence, there exists $N>0$ satisfying

$$
\zeta_{k} \in(-N, 0), \quad k \geq L
$$

In fact, it suffices to put

$$
N:=\min \left\{\zeta_{L}, \zeta_{L+1}, \ldots, \zeta_{l_{0}}, X_{2}\right\}
$$


Trivially, from (3.22) and (3.23) (or directly from (3.14)), it is seen that

$$
\left|\Delta \zeta_{k}\right| \leq \frac{1}{k-p+2}\left[(p-1) N+s^{+}+\frac{2^{q-1}(p-1) N^{q}}{\left[r^{-}\right]^{q-1}}\right], \quad k \geq L .
$$

Therefore, there exists $P>0$ for which

$$
\left|\Delta \zeta_{k}\right|<\frac{P}{k}, \quad k \geq L
$$

Especially, (3.29) gives $Q>0$ such that

$$
\left|\zeta_{k+i}-\zeta_{k+j}\right|<\frac{Q}{k}, \quad i, j \in\{0, \ldots, m-1\}, k \geq L .
$$

Indeed (consider (3.28)), inequalities (3.29) and (3.30) are valid for

$$
\begin{aligned}
P & :=\sup _{k \in \mathbb{N}_{L}} \frac{k}{k-p+2}\left[(p-1) N+s^{+}+\frac{2^{q-1}(p-1) N^{q}}{\left[r^{-}\right]^{q-1}}\right] \\
& =\max \left\{1, \frac{L}{L-p+2}\right\}\left[(p-1) N+s^{+}+\frac{2^{q-1}(p-1) N^{q}}{\left[r^{-}\right]^{q-1}}\right]
\end{aligned}
$$

and $Q:=(m-1) P$.

In addition (see Definition 2), we can assume that $m \in \mathbb{N}$ from (3.4) is so large that

$$
\frac{1}{m+j} \sum_{k=i}^{i+m+j-1} s_{k}>q^{-p}\left(\frac{1}{m+l} \sum_{k=i}^{i+m+l-1} r_{k}^{1-q}\right)^{1-p}, \quad i \in \mathbb{N}_{a}, j, l \in \mathbb{N} \cup\{0\} .
$$

To obtain the oscillatory part of the theorem, one can proceed as in the proof of Theorem 4.1 in [5], where only (3.4), (3.17), (3.18), (3.27), (3.30), and (3.31) are used to get a contradiction with the existence of the negative solution $\left\{\zeta_{k}\right\}_{k \in \mathbb{N}_{L}}$ (in fact, these inequalities are used only in special forms therein).

In the non-oscillatory part of the proof, we consider that $m \in \mathbb{N}$ satisfies

$$
\frac{1}{m+j} \sum_{k=i}^{i+m+j-1} s_{k}<q^{-p}\left(\frac{1}{m+l} \sum_{k=i}^{i+m+l-1} r_{k}^{1-q}\right)^{1-p}, \quad i \in \mathbb{N}_{a}, j, l \in \mathbb{N} \cup\{0\} .
$$

Let $\left\{\zeta_{k}\right\}_{k \in \mathbb{N}_{L}}$ be the solution of the Cauchy problem given by (2.12) and

$$
\zeta_{L}=-\left(\frac{q}{m} \sum_{i=L}^{L+m-1} r_{i}^{1-q}\right)^{1-p}
$$

where $L \in \mathbb{N}$ is sufficiently large. As in the first part of the proof, we obtain (3.22) and (3.23) if $\zeta_{k}$ is negative. Thus, we can assume that $L$ is so large that

$$
2 \zeta_{L}<\zeta_{L+i}<0, \quad i \in\{1, \ldots, m\}
$$

In addition (compare (3.33) with (3.27)), as in the first part, one can estimate

$$
\left|\Delta \zeta_{L+i}\right|<\frac{\widetilde{P}}{L}, \quad i \in\{0,1, \ldots, m-1\},
$$




$$
\left|\zeta_{L+i}-\zeta_{L+j}\right|<\frac{\widetilde{Q}}{L}, \quad i, j \in\{0,1, \ldots, m\}
$$

for some $\widetilde{P}, \widetilde{Q}>0$. Now the process from the proof of Theorem 4.1 in [5] shows that $\zeta_{k}$ is negative for all $k \geq L$, because only (3.4), (3.17), (3.18), (3.32), (3.34), and (3.35) are used therein. Hence, to complete the proof, it suffices to apply Lemma 3.

We slightly improve Theorem 5 into the following form (more common in the literature). In particular, we remove the requirement on sequence $\left\{s_{k}\right\}$ that it has a positive mean value.

Theorem 6 Let us consider the equation

$$
\Delta\left[\tilde{r}_{k} \Phi\left(\Delta x_{k}\right)\right]+\frac{\tilde{s}_{k}}{(k+1)^{(p)}} \Phi\left(x_{k+1}\right)=0
$$

where the coefficients $\left\{\tilde{r}_{k}\right\}_{k \in \mathbb{N}_{a}},\left\{\tilde{s}_{k}\right\}_{k \in \mathbb{N}_{a}}$ be such that the mean values of sequences $\left\{\tilde{r}_{k}^{1-q}\right\}$, $\left\{\tilde{s}_{k}\right\}$ exist and $\left\{\tilde{r}_{k}\right\}$ is bounded and positive. Let us denote

$$
\Gamma:=q^{-p}\left[M\left(\left\{\tilde{r}_{k}^{1-q}\right\}\right)\right]^{1-p} .
$$

Equation (3.36) is oscillatory if $M\left(\left\{\tilde{s}_{k}\right\}\right)>\Gamma$. Equation (3.36) is non-oscillatory if $M\left(\left\{\tilde{s}_{k}\right\}\right)<$ $\Gamma$.

Proof Considering Lemma 1 for $\left\{\tilde{r}_{k}^{1-q}\right\}$ and the boundedness of $\left\{\tilde{r}_{k}\right\}$, we know that

$$
0<\inf _{k \in \mathbb{N}_{a}} \tilde{r}_{k}^{1-q} \leq \sup _{k \in \mathbb{N}_{a}} \tilde{r}_{k}^{1-q}<\infty, \quad \text { i.e., } \infty>\sup _{k \in \mathbb{N}_{a}} \tilde{r}_{k} \geq \inf _{k \in \mathbb{N}_{a}} \tilde{r}_{k}>0 .
$$

We use Theorem 5. Therefore, we assume that $M\left(\left\{\tilde{s}_{k}\right\}\right)>0$.

We divide (3.36) by the constant value $\left[M\left(\left\{\tilde{r}_{k}^{1-q}\right\}\right)\right]^{1-p}>0$ (see (3.38)). We obtain the equation

$$
\Delta\left[\frac{\tilde{r}_{k} \Phi\left(\Delta x_{k}\right)}{\left[M\left(\left\{\tilde{r}_{k}^{-q}\right\}\right)\right]^{1-p}}\right]+\frac{\tilde{s}_{k} \Phi\left(x_{k+1}\right)}{(k+1)^{(p)}\left[M\left(\left\{\tilde{r}_{k}^{-q}\right\}\right)\right]^{1-p}}=0
$$

which has the form of (2.9) with the coefficients

$$
r_{k}=\frac{\tilde{r}_{k}}{\left[M\left(\left\{\tilde{r}_{k}^{-q}\right\}\right)\right]^{1-p}}, \quad s_{k}=\frac{\tilde{s}_{k}}{\left[M\left(\left\{\tilde{r}_{k}^{1-q}\right\}\right)\right]^{1-p}} .
$$

Especially, we have

$$
M\left(\left\{r_{k}^{1-q}\right\}\right)=M\left(\left\{\frac{\tilde{r}_{k}^{1-q}}{\left[M\left(\left\{\tilde{r}_{k}^{1-q}\right\}\right)\right]^{(1-p)(1-q)}}\right\}\right)=\left[M\left(\left\{\tilde{r}_{k}^{1-q}\right\}\right)\right]^{-1} M\left(\left\{\tilde{r}_{k}^{1-q}\right\}\right)=1,
$$

where the identity

$$
(1-p)(1-q)=1
$$

is used. 
According to Theorem 5, (3.39) is oscillatory if

$$
M\left(\left\{s_{k}\right\}\right)=M\left(\left\{\frac{\tilde{s}_{k}}{\left[M\left(\left\{\tilde{r}_{k}^{1-q}\right\}\right)\right]^{1-p}}\right\}\right)=\left[M\left(\left\{\tilde{r}_{k}^{1-q}\right\}\right)\right]^{p-1} M\left(\left\{\tilde{s}_{k}\right\}\right)>q^{-p},
$$

and non-oscillatory if

$$
M\left(\left\{s_{k}\right\}\right)=\left[M\left(\left\{\tilde{r}_{k}^{1-q}\right\}\right)\right]^{p-1} M\left(\left\{\tilde{s}_{k}\right\}\right)<q^{-p} .
$$

This fact implies the statement of Theorem 6 for any positive mean value of $\left\{\tilde{s}_{k}\right\}$.

Now, let $M\left(\left\{\tilde{s}_{k}\right\}\right) \leq 0$. Then there exists a positive constant $C$ such that

$$
0<M\left(\left\{\tilde{s}_{k}\right\}\right)+C=M\left(\left\{\tilde{s}_{k}+C\right\}\right)<\Gamma .
$$

We consider the non-oscillatory equation

$$
\Delta\left[\tilde{r}_{k} \Phi\left(\Delta x_{k}\right)\right]+\frac{\tilde{s}_{k}+C}{(k+1)^{(p)}} \Phi\left(x_{k+1}\right)=0,
$$

which is a majorant equation of (3.36). Thus, the proof can be completed by the application of Theorem 3.

Since the presented results are new also for linear difference equations (the case that $p=q=2$ ), we mention the following direct corollary of Theorem 6 .

\section{Corollary 1 Let us consider the equation}

$$
\Delta\left[r_{k} \Delta x_{k}\right]+\frac{s_{k} x_{k+1}}{(k+1) k}=0,
$$

where the sequences $\left\{r_{k}\right\}_{k \in \mathbb{N}_{a}}$ and $\left\{s_{k}\right\}_{k \in \mathbb{N}_{a}}$ have the properties that $M\left(\left\{r_{k}^{-1}\right\}\right)$ and $M\left(\left\{s_{k}\right\}\right)$ exist and $\left\{r_{k}\right\}$ is bounded and positive. Then (3.41) is oscillatory for

$$
M\left(\left\{r_{k}^{-1}\right\}\right) M\left(\left\{s_{k}\right\}\right)>\frac{1}{4}
$$

and non-oscillatory for

$$
M\left(\left\{r_{k}^{-1}\right\}\right) M\left(\left\{s_{k}\right\}\right)<\frac{1}{4}
$$

Based on results of [24] (see also $[25,26]$ ), the conjecture is given in our previous paper [5] that the border case $M\left(\left\{r_{k}^{-1}\right\}\right) M\left(\left\{s_{k}\right\}\right)=1 / 4$ from Corollary 1 is not solvable for general coefficients; i.e., in the border case, there exist oscillatory equations in the form of (3.41) and, at the same time, there exist non-oscillatory equations in this form.

In addition, using the Sturm type comparison theorem, we get the next new result concerning non-oscillatory half-linear difference equations when the coefficient in the difference term does not need to be bounded.

Theorem 7 Let us consider (3.36) and $\Gamma$ introduced in (3.37). Let the coefficients $\left\{\tilde{r}_{k}\right\}_{k \in \mathbb{N}_{a}}$, $\left\{\tilde{s}_{k}\right\}_{k \in \mathbb{N}_{a}}$ be such that the mean values of sequences $\left\{\tilde{r}_{k}^{1-q}\right\},\left\{\tilde{s}_{k}\right\}$ exist and $\left\{\tilde{r}_{k}\right\}$ is positive. Then (3.36) is non-oscillatory if $M\left(\left\{\tilde{s}_{k}\right\}\right)<\Gamma$. 
Proof The inequality $M\left(\left\{\tilde{s}_{k}\right\}\right)<\Gamma$ can be trivially rewritten into the form

$$
M\left(\left\{\tilde{s}_{k}\right\}\right)<q^{-p}\left[M\left(\left\{\tilde{r}_{k}^{1-q}\right\}\right)\right]^{1-p}-\delta
$$

for some $\delta>0$. In particular, there exists $\vartheta>0$ for which

$$
M\left(\left\{\tilde{s}_{k}\right\}\right)<q^{-p}\left[M\left(\left\{\tilde{r}_{k}^{1-q}\right\}\right)+\vartheta\right]^{1-p} .
$$

From Definition 2 and (3.40), it is seen that the sequence $\left\{\tilde{R}_{k}\right\}_{k \in \mathbb{N}_{a}}$ given by

$$
\tilde{R}_{k}:=\left(\tilde{r}_{k}^{1-q}+\vartheta\right)^{1-p}, \quad k \in \mathbb{N}_{a}
$$

satisfies

$$
M\left(\left\{\tilde{R}_{k}^{1-q}\right\}\right)=M\left(\left\{\tilde{r}_{k}^{1-q}+\vartheta\right\}\right)=M\left(\left\{\tilde{r}_{k}^{1-q}\right\}\right)+\vartheta>0 .
$$

In addition, sequence $\left\{\tilde{R}_{k}\right\}$ is bounded. Thus, we can apply Theorem 6 which guarantees that the equation

$$
\Delta\left[\tilde{R}_{k} \Phi\left(\Delta x_{k}\right)\right]+\frac{\tilde{s}_{k}}{(k+1)^{(p)}} \Phi\left(x_{k+1}\right)=0
$$

is non-oscillatory (see (3.43) and (3.44)). Of course, (3.45) is a majorant of (3.36) because $\tilde{R}_{k} \leq \tilde{r}_{k}$ for all considered $k$ (see again (3.40)). Finally, it suffices to use Theorem 3 .

Again, from the theorem above, we obtain a new result in the linear case. The linear version of Theorem 7 reads as follows.

Corollary 2 Let us consider (3.41) with the coefficients $\left\{r_{k}\right\}_{k \in \mathbb{N}_{a}}$ and $\left\{s_{k}\right\}_{k \in \mathbb{N}_{a}}$ such that $M\left(\left\{r_{k}^{-1}\right\}\right)$ and $M\left(\left\{s_{k}\right\}\right)$ exist and $\left\{r_{k}\right\}$ is positive. Then (3.41) is non-oscillatory if (3.42) is valid.

\section{Examples}

In this section, we give some simple examples of oscillatory and non-oscillatory equations whose oscillatory properties do not follow from any previously known oscillation or non-oscillation criteria. To illustrate Theorems 5, 6, 7 and Corollaries 1, 2, we mention Examples 1, 2, 4 and Examples 3, 5, respectively.

Example 1 Let $a, b>0$ be arbitrary. The equation

$$
\Delta\left[\Phi\left(\Delta x_{k}\right)\right]+\frac{a|\sin k|+b \cos k}{(k+1)^{(p)}} \Phi\left(x_{k+1}\right)=0
$$

has evidently the form of (2.9). Since

$$
M(\{a|\sin k|+b \cos k\})=a M(\{|\sin k|\})+b M(\{\cos k\})=\frac{2 a}{\pi},
$$

(4.1) is oscillatory for $2 a>q^{-p} \pi$ and non-oscillatory for $2 a<q^{-p} \pi$. 
Example 2 Let $\lambda, \mu \in \mathbb{R}$ be arbitrarily given, where $|\mu|>|\lambda|$. Let us consider the equation

$$
\Delta\left[\left|\sin \frac{(3 k+1) \pi}{9}\right|^{-\frac{p}{q}} \Phi\left(\Delta x_{k}\right)\right]+\frac{\lambda+(-1)^{k} \mu}{k^{(p)}} \Phi\left(x_{k+1}\right)=0,
$$

which has the form of (3.36) for

$$
\tilde{r}_{k}=\left|\sin \frac{(3 k+1) \pi}{9}\right|^{-\frac{p}{q}}, \quad \tilde{s}_{k}=\left[\lambda+(-1)^{k} \mu\right] \frac{(k+1)^{(p)}}{k^{(p)}} .
$$

Since

$$
\begin{aligned}
M\left(\left\{\tilde{r}_{k}^{1-q}\right\}\right) & =M\left(\left\{\left|\sin \frac{(3 k+1) \pi}{9}\right|\right\}\right)=\frac{1}{6} \sum_{k=1}^{6}\left|\sin \frac{(3 k+1) \pi}{9}\right| \\
& =\frac{1}{3}\left(\sin \frac{\pi}{9}+\sin \frac{2 \pi}{9}+\sin \frac{4 \pi}{9}\right)=\frac{2}{3}\left(\sin \frac{\pi}{9}+\sin \frac{2 \pi}{9}\right)
\end{aligned}
$$

and

$$
M\left(\left\{\tilde{s}_{k}\right\}\right)=M\left(\left\{\lambda+(-1)^{k} \mu\right\}\right)=\lambda,
$$

considering Theorem 6, we know that (4.2) is oscillatory for

$$
\lambda>\Gamma:=q^{-p}\left[\frac{2}{3}\left(\sin \frac{\pi}{9}+\sin \frac{2 \pi}{9}\right)\right]^{1-p}
$$

and non-oscillatory for $\lambda<\Gamma$.

Example 3 Let $K_{1}, L_{1}, K_{2}, L_{2}>0$. We define the sequence $\left\{r_{k}\right\}_{k \in \mathbb{N}}$ by the formula

$$
r_{k}:= \begin{cases}K_{1}+L_{1}, & k \in\left\{2^{n} ; n \in \mathbb{N}\right\} ; \\ K_{1}, & k \in \mathbb{N} \backslash\left\{2^{n} ; n \in \mathbb{N}\right\},\end{cases}
$$

and the sequence $\left\{s_{k}\right\}_{k \in \mathbb{N}}$ by

$$
s_{k}:= \begin{cases}K_{2}+(-1)^{n} L_{2}, & k \in\left\{3^{n} ; n \in \mathbb{N}\right\} ; \\ K_{2}, & k \in \mathbb{N} \backslash\left\{3^{n} ; n \in \mathbb{N}\right\} .\end{cases}
$$

If we consider these functions as the coefficients in (3.41), then this equation is oscillatory for

$$
M\left(\left\{r_{k}^{-1}\right\}\right) M\left(\left\{s_{k}\right\}\right)=\frac{K_{2}}{K_{1}}>\frac{1}{4}
$$

and non-oscillatory for $K_{1}>4 K_{2}$. Indeed, we can apply Corollary 1.

Example 4 Let $\gamma>0$. We use Theorem 7 for the following equation:

$$
\Delta\left[\frac{1}{1+\cos k \cdot \sin (\sqrt{2} k)} \cdot \frac{\Delta x_{k}}{\sqrt{\left|\Delta x_{k}\right|}}\right]+\frac{1}{\gamma \sqrt{k^{3}}} \cdot \frac{x_{k+1}}{\sqrt{\left|x_{k+1}\right|}}=0,
$$


where $p=3 / 2($ i.e., $q=3$ ) and

$$
\tilde{r}_{k}=\frac{1}{1+\cos k \cdot \sin (\sqrt{2} k)}, \quad \tilde{s}_{k}=\frac{(k+1)^{(3 / 2)}}{\gamma \sqrt{k^{3}}}
$$

for all large $k \in \mathbb{N}$. One can easily verify that

$$
\begin{aligned}
M\left(\left\{\tilde{r}_{k}^{-2}\right\}\right) & =M\left(\left\{[1+\cos k \cdot \sin (\sqrt{2} k)]^{2}\right\}\right) \\
& =1+M\left(\left\{\cos ^{2} k \cdot \sin ^{2}(\sqrt{2} k)\right\}\right)=\frac{5}{4}
\end{aligned}
$$

and that

$$
M\left(\left\{\tilde{s}_{k}\right\}\right)=\frac{1}{\gamma} \lim _{k \rightarrow \infty} \frac{(k+1)^{(3 / 2)}}{\sqrt{k^{3}}}=\frac{1}{\gamma} .
$$

Thus, (4.3) is non-oscillatory if $2 \gamma>3 \sqrt{15}$.

Example 5 For any $c<1 / 4$, the linear equation

$$
\Delta\left[\frac{\Delta x_{k}}{1+\cos k^{2}}\right]+\frac{c}{(k+1) k} x_{k+1}=0
$$

satisfies all assumptions of Corollary 2. It is seen that

$$
M\left(\left\{r_{k}^{-1}\right\}\right) M\left(\left\{s_{k}\right\}\right)=M\left(\left\{1+\cos k^{2}\right\}\right) M(\{c\})=c<\frac{1}{4},
$$

which means that (4.4) is non-oscillatory.

Now we briefly explain why the oscillatory problems in the above examples are not covered by any previously known results (see also Theorem 1). In both of Examples 1 and 2, the second coefficient changes its sign. In Example 3, the coefficients are not asymptotically almost periodic. In Example 4, the coefficient in the difference term is not bounded. In the last example, the first coefficient is not asymptotically almost periodic and, at the same time, it is not bounded.

As a final remark, we focus our attention on the denominators of the potentials considered in Examples 2 and 4, where $(k+1)^{(p)}$ and $(k+1)^{(3 / 2)}$ is replaced by $k^{(p)}$ and $\sqrt{k^{3}}$, respectively. In fact, all presented results remain true if we replace the coefficients $\left\{s_{k}\right\}$ or $\left\{\tilde{s}_{k}\right\}$ by $\left\{f_{k} \cdot s_{k}\right\}$ or $\left\{f_{k} \cdot \tilde{s}_{k}\right\}$ for any sequence of real numbers $f_{k}$ satisfying $\lim _{k \rightarrow \infty} f_{k}=1$. Indeed, the existence of $M\left(\left\{h_{k}\right\}\right)$ implies that $M\left(\left\{h_{k} \cdot g_{k}\right\}\right)=M\left(\left\{h_{k}\right\}\right)$ whenever $\lim _{k \rightarrow \infty} g_{k}=1$ (consider Definition 2 and Lemma 1). Note that we consider the denominator $(k+1)^{(p)}$ due to the form of previously known results (see Section 1).

Competing interests

The authors declare that they have no competing interests.

Authors' contributions

The authors declare that the research was realized in collaboration with the same responsibility and contributions. All authors read and approved the final manuscript. 


\section{Acknowledgements}

The first author is supported by Grant P201/10/1032 of the Czech Science Foundation. The second author is supported by the project 'Employment of Best Young Scientists for International Cooperation Empowerment'

(CZ.1.07/2.3.00/30.0037) co-financed from European Social Fund and the state budget of the Czech Republic.

Received: 25 February 2015 Accepted: 17 June 2015 Published online: 09 July 2015

\section{References}

1. Kelley, WG, Peterson, AC: Difference Equations: An Introduction with Applications. Academic Press, San Diego (2001)

2. Naiman, PB: The set of isolated points of increase of the spectral function pertaining to a limit-constant Jacobi matrix Izv. Vysš. Učebn. Zaved., Mat. 1(8), 129-135 (1959) (in Russian)

3. Luef, F, Teschl, G: On the finiteness of the number of eigenvalues of Jacobi operators below the essential spectrum. J. Differ. Equ. Appl. 10(3), 299-307 (2004) doi:10.1080/10236190310001641227

4. Hasil, P, Veselý, M: Critical oscillation constant for difference equations with almost periodic coefficients. Abstr. Appl. Anal. 2012, Article ID 471435 (2012). doi:10.1155/2012/471435

5. Veselý, M, Hasil, P: Oscillation and non-oscillation of asymptotically almost periodic half-linear difference equations. Abstr. Appl. Anal. 2013, Article ID 432936 (2013). doi:10.1155/2013/432936

6. Kneser, A: Untersuchungen über die reellen Nullstellen der Integrale linearer Differentialgleichungen. Math. Ann. 42(3), 409-435 (1893). doi:10.1007/BF01444165

7. Gesztesy, F, Ünal, M: Perturbative oscillation criteria and Hardy-type inequalities. Math. Nachr. 189, 121-144 (1998). doi:10.1002/mana.19981890108

8. Schmidt, KM: Oscillation of perturbed Hill equation and lower spectrum of radially periodic Schrödinger operators in the plane. Proc. Am. Math. Soc. 127(8), 2367-2374 (1999). doi:10.1090/S0002-9939-99-05069-8

9. Krüger, H: On perturbations of quasiperiodic Schrödinger operators. J. Differ. Equ. 249(6), 1305-1321 (2010). doi:10.1016/j.jde.2010.06.018

10. Krüger, H, Teschl, G: Effective Prüfer angles and relative oscillation criteria. J. Differ. Equ. 245(12), 3823-3848 (2008). doi:10.1016/j.jde.2008.06.004

11. Krüger, H, Teschl, G: Relative oscillation theory for Sturm-Liouville operators extended. J. Funct. Anal. 254(6), 1702-1720 (2008). doi:10.1016/j.jfa.2007.10.007

12. Schmidt, KM: Critical coupling constant and eigenvalue asymptotics of perturbed periodic Sturm-Liouville operators. Commun. Math. Phys. 211(2), 465-485 (2000). doi:10.1007/s002200050822

13. Elbert, Á: Oscillation and nonoscillation theorems for some nonlinear ordinary differential equations. In: Ordinary and Partial Differential Equations (Dundee, 1982). Lecture Notes in Math., vol. 964, pp. 187-212. Springer, Berlin (1982)

14. Elbert, Á: Asymptotic behaviour of autonomous half-linear differential systems on the plane. Studia Sci. Math. Hung. 19(2-4), 447-464 (1984)

15. Hasil, P: Conditional oscillation of half-linear differential equations with periodic coefficients. Arch. Math. 44(2), 119-131 (2008)

16. Došlý, O, Funková, H: Euler type half-linear differential equation with periodic coefficients. Abstr. Appl. Anal. 2013, Article ID 714263 (2013). doi:10.1155/2013/714263

17. Došlý, O, Hasil, P: Critical oscillation constant for half-linear differential equations with periodic coefficients. Ann. Mat. Pura Appl. 190(3), 395-408 (2011). doi:10.1007/s10231-010-0155-0

18. Hasil, P, Veselý, M: Oscillation of half-linear differential equations with asymptotically almost periodic coefficients. Adv. Differ. Equ. 2013, 122 (2013). doi:10.1186/1687-1847-2013-122

19. Veselý, M, Hasil, P: Conditional oscillation of Riemann-Weber half-linear differential equations with asymptotically almost periodic coefficients. Studia Sci. Math. Hung. 51(3), 303-321 (2014). doi:10.1556/SScMath.51.2014.3.1283

20. Veselý, M, Hasil, P, Mařík, R: Conditional oscillation of half-linear differential equations with coefficients having mean values. Abstr. Appl. Anal. 2014, Article ID 258159 (2014). doi:10.1155/2014/258159

21. Agarwal, RP, Bohner, M, Grace, SR, O'Regan, D: Discrete Oscillation Theory. Hindawi Publishing Corporation, New York (2005)

22. Došlý, O, Řehák, P: Half-Linear Differential Equations. Elsevier, Amsterdam (2005)

23. Řehák, P: Hartman-Wintner type lemma, oscillation, and conjugacy criteria for half-linear difference equations. J. Math. Anal. Appl. 252(2), 813-827 (2000). doi:10.1006/jmaa.2000.7124

24. Veselý, M: Construction of almost periodic sequences with given properties. Electron. J. Differ. Equ. 2008, 126 (2008)

25. Veselý, M: Almost periodic homogeneous linear difference systems without almost periodic solutions. J. Differ. Equ. Appl. 18(10), 1623-1647 (2012). doi:10.1080/10236198.2011.585984

26. Veselý, M: Construction of almost periodic functions with given properties. Electron. J. Differ. Equ. 2011, 29 (2011) 